\title{
Modeling Intense Beam Propagation in the Paul Trap Simulator Experiment (PTSX)
}

\author{
Erik P. Gilson, Ronald C. Davidson, Philip C. Efthimion, Richard Majeski, \\ and Edward A. Startsev
}

Plasma Physics Laboratory, Princeton University, Princeton, New Jersey, 08543

\begin{abstract}
The Paul Trap Simulator Experiment (PTSX) is a compact laboratory facility whose purpose is to simulate the nonlinear dynamics of intense charged particle beam propagation over large distances through an alternating-gradient magnetic transport system. The PTSX device is a $200 \mathrm{~cm}$ long, $20 \mathrm{~cm}$ diameter cylindrical Paul trap in which a $400 \mathrm{~V}, 100 \mathrm{kHz}$ signal confines cesium ions to an rms radius of $1 \mathrm{~cm}$. The one-component cesium plasmas can be confined for hundreds of milliseconds, which would correspond to an equivalent alternating-gradient transport system many kilometers long. The normalized intensity parameter $\hat{s}=\left.\omega_{p}^{2}\right|_{r=0} / 2 \omega_{q}^{2}$, where $\omega_{q}$ is the average transverse focusing frequency, describes whether the plasma is emittance-dominated $(\hat{s} \ll 1)$ or space-charge-dominated $(\hat{s} \rightarrow 1)$. By increasing the amount of charge loaded into the trap, PTSX reaches values of $\hat{s}=0.8$. Thus, the opportunity exists to study important physics topics such as: the conditions necessary for quiescent intense beam propagation over large distances, beam mismatch and envelope instabilities, collective mode excitations, the dynamics and production of halo particles, emittance growth, compression techniques, and the effects of the distribution function on stability properties. Results are presented that demonstrate the sustainment of the radial density profile over long times, and the ability of PTSX to reach large $\hat{s}$. The results of initial threedimensional particle-in-cell simulations are also presented.
\end{abstract}

\section{INTRODUCTION}

The Paul Trap Simulator Experiment (PTSX) is a cylindrical Paul trap whose purpose is to simulate the transverse dynamics of charged particle beams in alternating-gradient (AG) magnetic transport systems [1]. The PTSX device is able to simulate such charged particle beam transport systems because the transverse equations of motion including both the externally applied forces and the self-field forces are similar as long as the beam radius $r_{b}$ is small compared to the spatial periodicity of the AG system $[1,2]$. This is because the magnetic forces that beam particles feel in the laboratory frame are Lorentz transformed into oscillatory electric forces in the beam frame. Because of the long confinement times of ions in PTSX relative to the oscillation frequency of the trap voltage, PTSX is capable of simulating the beam dynamics over equivalent propagation distances of many kilometers. Perhaps most importantly, the waveform of the trap voltage is controlled by an arbitrary function generator so that the waveform can be varied in order to study a wide variety of focusing field configurations.

The novel, essential feature of PTSX is its ability to study intense beams in which the space-charge is non-negligible compared to the confining force, and the normalized intensity parameter $\hat{s}=\left.\omega_{p}^{2}\right|_{r=0} / 2 \omega_{q}^{2}$ [where $\omega_{p}(r)$ is the plasma frequency, and $\omega_{q}$ is the smooth focusing applied betatron frequency] approaches unity. Such intense beams 
are of increasing interest due to their relevance to high energy and nuclear physics, heavy ion fusion, spallation neutron sources, tritium production, and nuclear waste transmutation [3, 4]. As new accelerator facilities use beams with increasing spacecharge intensity, a basic understanding of the complex nonlinear interactions in the system becomes increasingly important. Our goal is to simulate the properties of highintensity beams where the self-field effects can significantly alter beam equilibrium, stability and transport properties.

In this paper, a brief description of the machine is presented. A reanalysis of previously presented data is shown to clarify the stability of the radial excursions of the particle trajectories. Data are presented that show the sustainment of the trapped plasma over long confinement times. Measurements demonstrating the ability of PTSX to reach intensities of $\hat{s}=0.8$ are shown. The initial results of 3D particle-in-cell simulations that aid in the interpretation of the data and help guide experimental planning are also presented. Finally, modifications that will improve the ion source performance and Faraday cup diagnostic are discussed.

\section{APPARATUS}

References 5 and 6 contain detailed descriptions of the PTSX device, and so only the essential concepts are summarized here. The PTSX device consists of three cylindrical electrodes of radius $r_{w}=10 \mathrm{~cm}$ that are sliced into four $90^{\circ}$ sectors as shown in Fig. 1. The central electrode has length $2 L=2 \mathrm{~m}$ while the end electrodes are each $40 \mathrm{~cm}$ long. The trap confines charged particles radially by applying a periodic voltage $\pm V_{0}(t)$ to the four sectors, creating a ponderomotive force that points radially inwards. A dc voltage $+\hat{V}$ applied to the end electrodes confines the ions axially. The ratio $L / r_{p}$ is large in order to minimize finite-length effects that are outside of the analogy. When the plasma radius $r_{p}$ is small compared to $r_{w}$, then the fields from the walls are almost purely quadrupolar with corrections on the order of $\left(r_{p} / r_{w}\right)^{4}$. For PTSX, $V_{0 \max } \leq 400 \mathrm{~V}, \hat{V}=150 \mathrm{~V}$, and the frequency $f$ of the oscillating voltage is less than $150 \mathrm{kHz}$. Presently, cesium atoms are used, although future experiments will use barium. With barium's atomic structure it will be straightforward to implement the laser-induced fluorescence (LIF) diagnostic planned for future experiments.

For the sinusoidal oscillations used to date, $\omega_{q}=4 e V_{0 \max } / \sqrt{2} m \pi^{2} r_{w}^{2} f$. Another important system parameter is the vacuum phase advance $\sigma_{v}$ that describes what portion of a transverse oscillation a beam particle completes per focusing system period. Thus, the smooth-focusing vacuum phase advance is given by $\sigma_{v}^{s f}=\omega_{q} / f$. Values from $0^{\circ}$ to beyond $90^{\circ}$ are easily accessible by the proper choice of $V_{0 \max }$ and $f$. For the experiments and simulations presented here, $V_{0 \text { max }}=235 \mathrm{~V}$ and $f=75 \mathrm{kHz}$, so that $\omega_{q}=6.51 \times 10^{4} \mathrm{~s}^{-1}$ and $\sigma_{v}^{s f}=49.7^{\circ}$.

The machine manipulates plasmas using a load—trap-dump cycle. During loading (dumping), the short electrodes on the source (diagnostic) end of the machine are made to oscillate with the same voltages $\pm V_{0}(t)$ as the long electrodes, which allows the ions to pass. When dumped, the ions strike a Faraday cup that has a collection aperture with a diameter, $2 r_{a p}=1 \mathrm{~cm}$. Either the charge or the average current can be measured with 

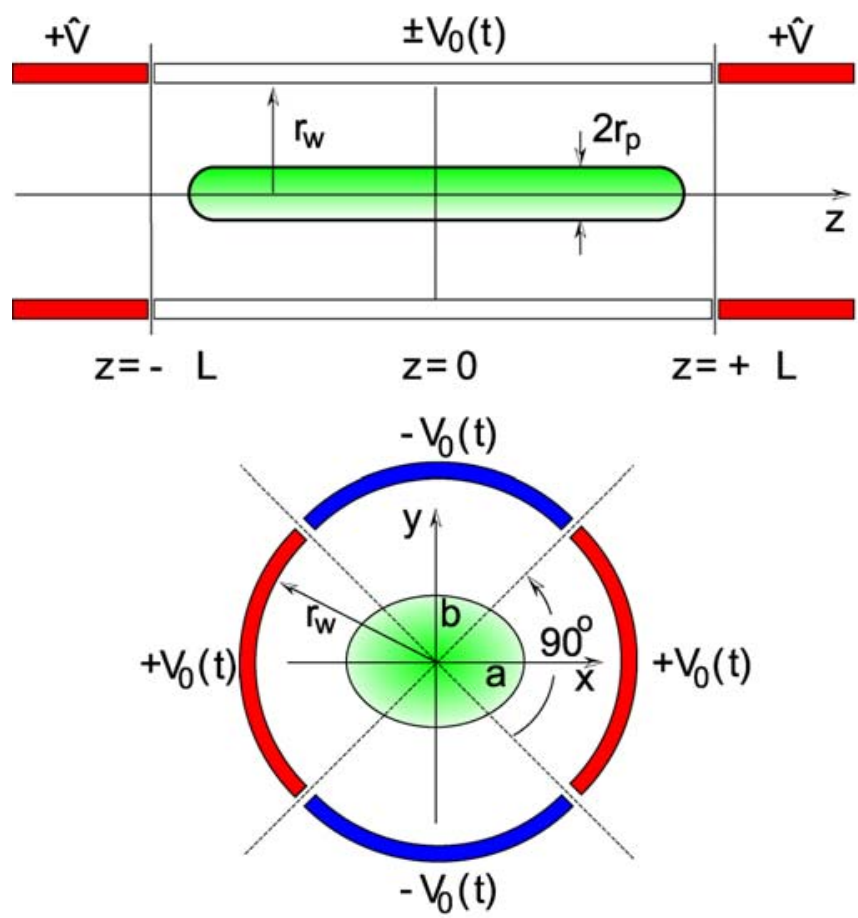

FIGURE 1. The PTSX device consists of three cylindrical electrodes with radius $r_{w}$, each sliced into four $90^{\circ}$ sectors. An oscillating voltage $\pm V_{0}(t)$ confines the plasma radially to a radius $r_{p}$. Static voltages $+\hat{V}$ on end electrodes confine the ions axially.

an electrometer to determine the radial plasma density profile.

\section{EXPERIMENTAL RESULTS}

Previously, it was reported that the single-particle orbits became unstable when the smooth-focusing vacuum phase advance $\sigma_{v}^{s f}$ exceeded $90^{\circ}$ [7]. This claim was based on the mistaken assumption that the solutions to the Mathieu equation became unstable at a phase advance of $\sigma_{v}=90^{\circ}$ and that the deviation of $\sigma_{v}^{s f}$ from the exact vacuum phase advance $\sigma_{v}$ was small. However, the solutions to the Mathieu become unstable at $\sigma_{v}=180^{\circ}$. Further, at such large phase advances, the difference between $\sigma_{v}$ and $\sigma_{v}^{s f}$ becomes large. By extending the analysis of Davidson and Qin [3], it is found that as $\sigma_{v}$ approaches $180^{\circ}, \sigma_{v}^{s f}$ approaches $115.6^{\circ}$ (see Fig. 2). Therefore, a more accurate description of the PTSX data in Ref. 7 is that the single-particle orbits become unstable when $\sigma_{v}=180^{\circ}$, or equivalently, when $\sigma_{v}^{s f}=115.6^{\circ}$. Since the difference between the curve for $\sigma_{v}^{s f}=90^{\circ}$ and $\sigma_{v}^{s f}=115.6^{\circ}$ is small, the error in the previously reported result is relatively small.

Radial density profiles are measured by dumping the plasma into a moveable Faraday 


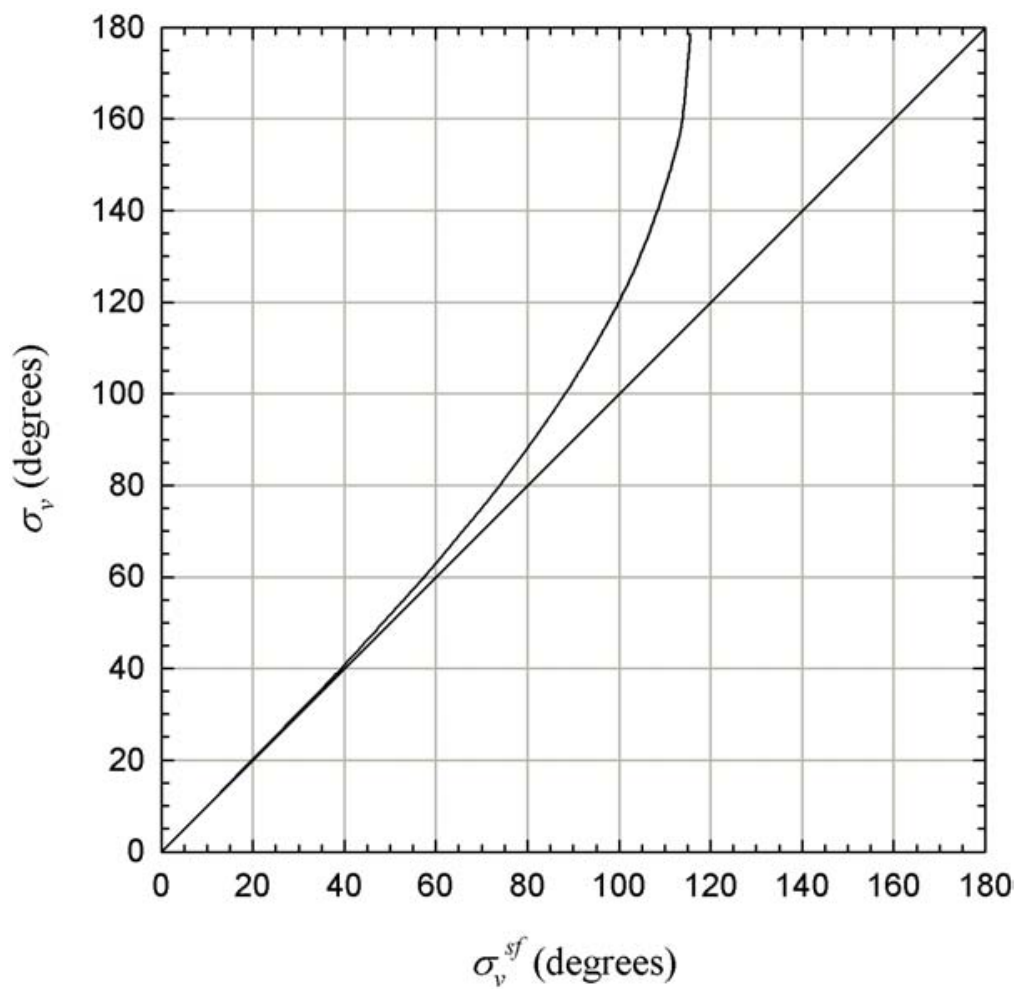

FIGURE 2. The exact vacuum phase advance $\sigma_{v}$ is obtained from numerically-determined matchedbeam solutions and compared with the phase advance $\sigma_{v}^{s f}$ determined using $\omega_{q}$ as calculated in the smooth-focusing approximation [3].

cup. Then, as described in Ref. 6, $r_{a p}$ and an estimate of the length of the plasma column $L_{p}$ allow the measured charge profile $Q(r)$ to be recast as a density profile $n(r)$. The estimate of $L_{p}$ is taken from particle-in-cell simulations (see below) and the resulting uncertainty in $L_{p}$ contributes a systematic uncertainty to quantities such as $N$ and $\hat{s}$. Since the amount of charge contained in the plasma is small $\left(n \leq 10^{6} \mathrm{~cm}^{-3}\right)$, the charges from hundreds or even thousands of "shots" of the experiment are averaged. The averaging reduces the effect of electrometer noise and allows better charge resolution.

Measurements of the radial profile as a function of hold time were presented in Ref. 6 where it was shown that the line charge $N=\int_{0}^{r_{w}} n(r) 2 \pi r d r$ remains relatively unchanged for hundreds of milliseconds. Since the PTSX electrodes oscillate with frequencies in the tens of kilohertz range, it is seen that PTSX can simulate beam transport over equivalent distances of many kilometers. Several of the individual radial profiles used to show the constancy of $N$ are shown in Fig. 3, including profiles corresponding to hold times of $1 \mathrm{~ms}, 10 \mathrm{~ms}$, and $100 \mathrm{~ms}$. It is interesting to note that the profile apparently becomes somewhat taller and narrower over time, while $N$ (given by the area under radial density profile) remains relatively constant. The profiles are reasonably described by Gaussians as is expected for moderately warm plasmas in thermal equilibrium [3]. Note that an offset of $0.62 \mathrm{~cm}$ has been removed from the profiles in Fig. 3 in order to center the 
peaks about $r=0 \mathrm{~cm}$. The origin of the offset is perhaps electrode asymmetries or distortion of the ion trajectories due to the Faraday cup.

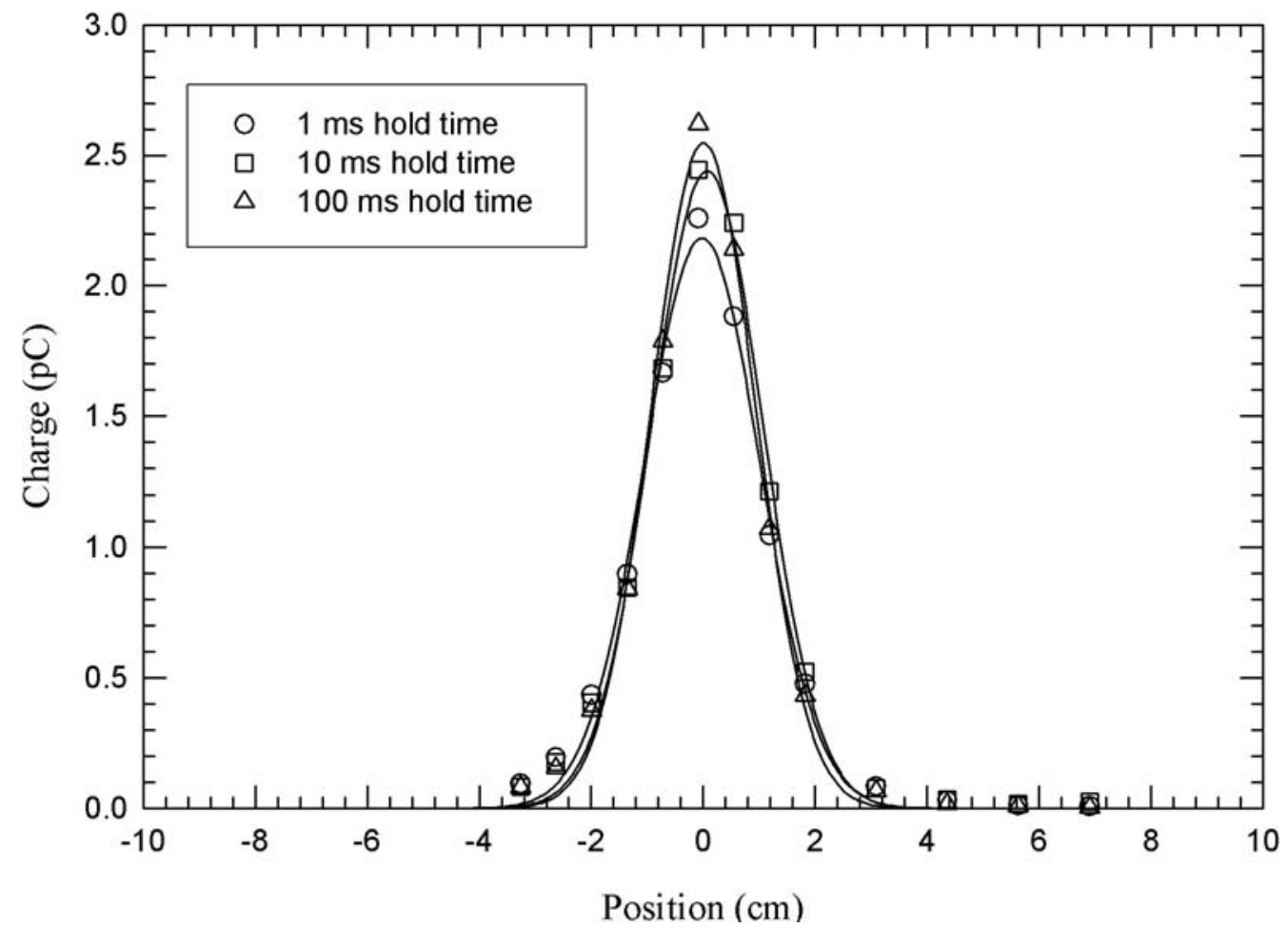

FIGURE 3. The radial profile of the plasma charge dumped into the Faraday cup is relatively unchanged after $100 \mathrm{~ms}$. For $f=75 \mathrm{kHz}$ and a spatial magnet period of $1 \mathrm{~m}$, this corresponds to beam transport over an equivalent distance of $7.5 \mathrm{~km}$.

An important feature of PTSX is the ability to perform experiments with plasmas for values of $\hat{s}$ approaching unity. By increasing the current injected into the trap, plasmas with $\hat{s} \leq 0.8$ have been observed. Figure 4 shows a radial profile for a plasma with $\hat{s}=0.8$. Note that the apparent halo structure is likely due to measurement noise since repeated measurements do not reliably reproduce this feature. As with Fig. 3, the data in Fig. 4 have had an offset removed, $0.72 \mathrm{~cm}$ in this case.

\section{PARTICLE-IN-CELL SIMULATIONS}

Three-dimensional particle-in-cell simulations employing the WARP-3D code [8] have been used to model the injection of plasmas into PTSX and investigate the properties of the trapped plasmas. The simulations include the detailed geometry of the ion source and the confinement electrodes. The code was run using a $48 \times 48 \times 512$ grid with $2.5 \times 10^{5}$ particles and a $0.66 \mu$ s timestep on 64 processors at NERSC. To compare a simulation to a set of experimental results, the simulation is started by adjusting the voltage on the acceleration grid to match the injection current in the simulation to that measured in the 


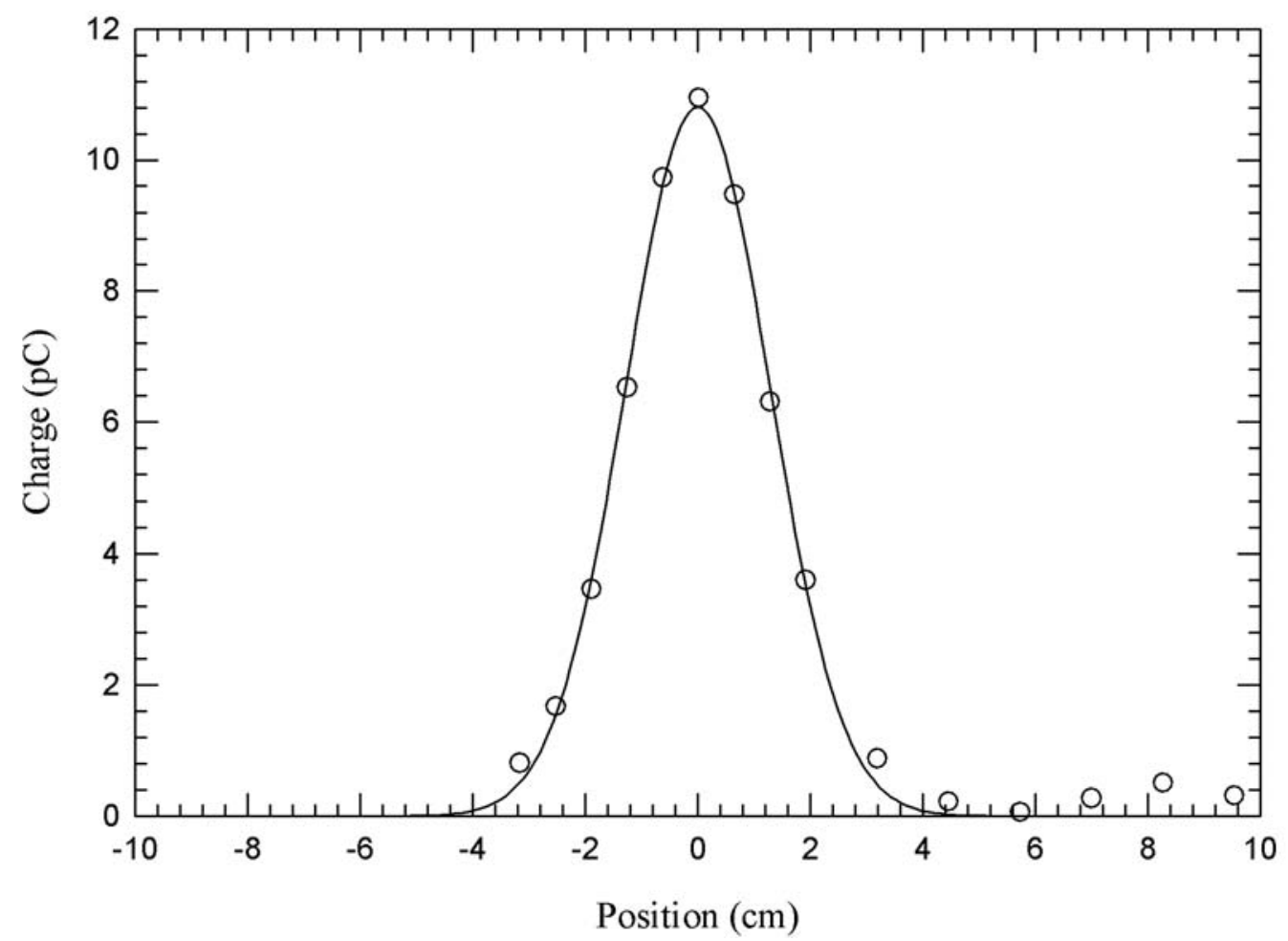

FIGURE 4. By increasing the current injected into the trap, the charge collected in the Faraday cup is increased and $\hat{s}=0.8$.

experiment. No other free parameters are necessary.

Simulations of the injection process are important because there is an inherent mismatch between the beam created at the ion source and the beam that we wish to simulate in PTSX. Matched beams have pulsating, elliptical cross sections that pulsate with the oscillation period of the transport system. The beam created at the ion source, however, has a circular cross section. Simulations confirm that this mismatch leads to some plasma heating and subsequent broadening of the plasma density profile.

Figure 5 shows the simulation particles and the equipotential surfaces in $(x, z)$ space immediately after the closure of the injection electrodes. An important result is an improved estimate of the plasma length $L_{p}$. To lowest order, one may assume that $L_{p}$ is the same as the length of the confinement electrodes, which is $200 \mathrm{~cm}$. However, the axial-trapping voltages $\hat{V}$ "leak" into the central trapping region as can be seen in Fig. 5. For a variety of parameters, it is found that $L_{p}$ is approximately $170 \mathrm{~cm}$. As evident from Fig. 5, the sudden change in the end-electrode potential in only one timestep has launched a density perturbation that propagates axially, back and forth. The Faraday cup cannot detect the presence or absence of this disturbance as it only measures $z$-averaged quantities. A simulation in which the trapping voltage is applied more gradually shows that the plasma is heated in the transverse direction rather than in the axial direction. 


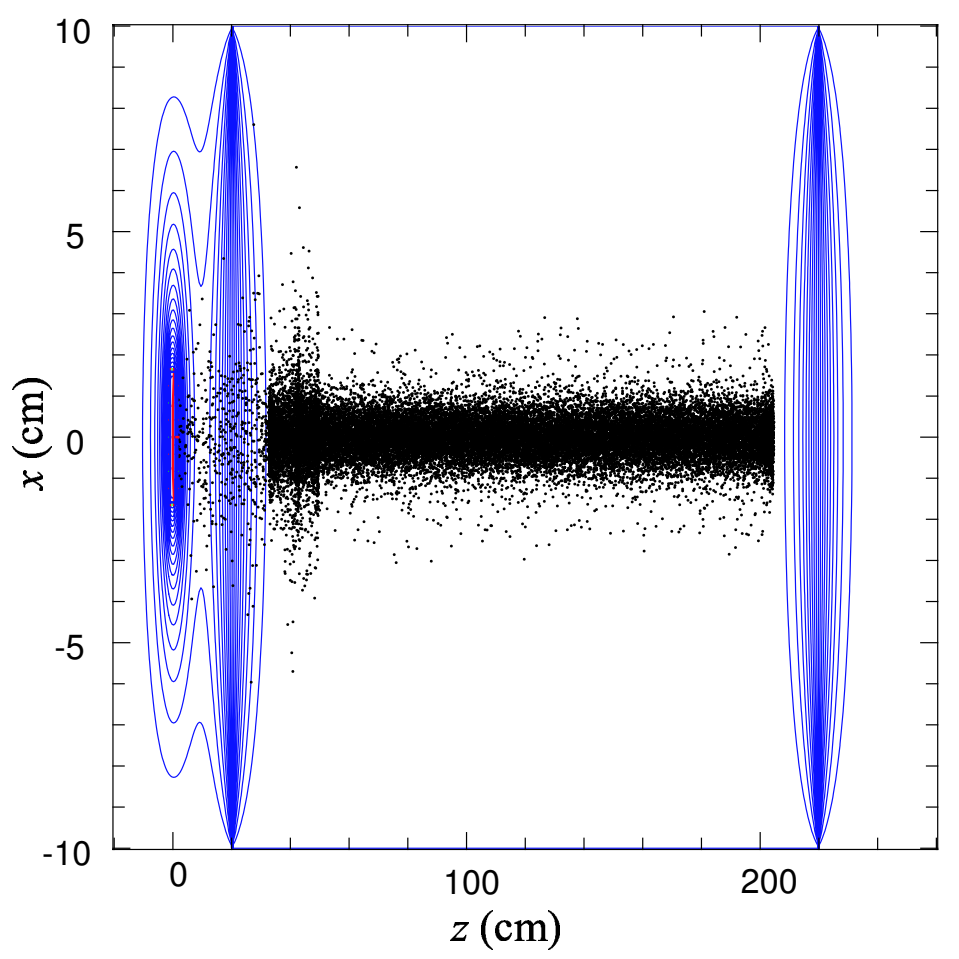

FIGURE 5. WARP 3D simulations showing the $(x, z)$ phase-space projection at $t=5.033 \mathrm{~ms}$, right after the closure of the injection electrodes at $t=5 \mathrm{~ms}$. Note that phase-space projections are generated every 50 timesteps. The equipotential contours exclude the transverse quadrupolar component and include only the self-field potential created by the ions and the applied end-electrode potentials.

\section{MACHINE MODIFICATIONS}

The two systems that are being improved in PTSX are the ion source system and the Faraday cup system. After adding a Pierce electrode to the original ion source design to inject smaller diameter plasmas, the ion source has been redesigned to ensure a geometry that is more nearly planar and to ensure azimuthal symmetry. Deviations from azimuthal symmetry lead to electric field perturbations that increase the transverse temperature of the injected plasma. In addition to the shielding added to the original Faraday cup system, a faceplate that spans the entire PTSX cross section now provides a conductive boundary that remains fixed even as the Faraday cup moves radially.

Engineering constraints required that the overall diameter of the previous ion source installation be 1.25 inches whereas the emitting surface itself is a 0.6 inch diameter disk. This allowed a Pierce electrode and grids where the difference between the outer radius and inner radius was only 0.325 inches. Moreover, electrical leads and grounded support structures passed through the Pierce electrode, degrading the azimuthal symmetry. By redesigning the ion source to be mounted directly on the vacuum-side of a large flange, transverse size constraints are removed and all source structures now extend to a diameter of four inches. The Pierce electrode and grids now open to a diameter of 2.75 inches 
(Fig. 6). The increased clearances allow for easier assembly and maintenance and for the addition of a type $\mathrm{K}$ thermocouple to the rear of the ion source. The thermocouple will allow the temperature and thus emission of the source to be better controlled.

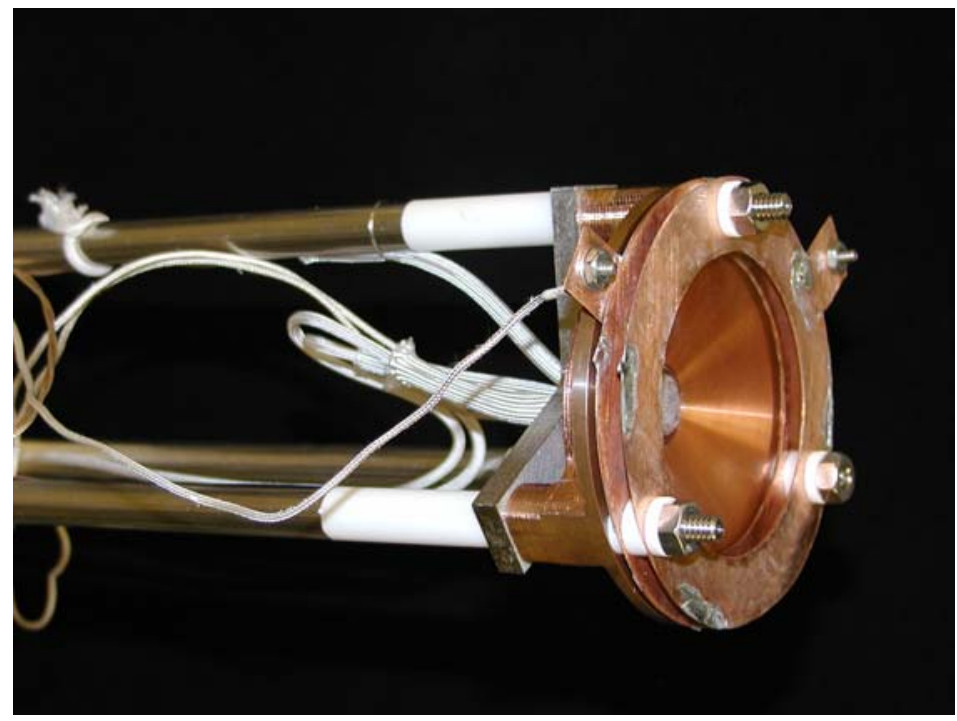

FIGURE 6. The ion source has an overall diameter of four inches while the grids and the conical surface of the Pierce electrode are 2.75 inches in diameter. The acceleration grid is placed $1 \mathrm{~cm}$ from the emitting surface and the spacing between the acceleration grid and deceleration grids is $0.5 \mathrm{~cm}$.

The previous Faraday cup design resulted in a variable boundary condition that changed as the Faraday cup was moved to different radial locations. The Faraday cup enclosure was a rectangular copper box that measured 1.25 inches by four inches. Thus, measurements of the radial density profile at the origin and at large radii occurred under different experimental conditions and may not be directly comparable. To ensure that the boundary condition instead remains fixed, the end of the dump electrodes has been fitted with a pair of copper disks (Fig. 7). The upstream disk has a slot (not visible in Fig. 7) that runs radially over three-quarters of the disk's diameter. The Faraday cup and its rectangular enclosure sit behind this disk so that the dumped plasma sees an equipotential boundary regardless of the position of the Faraday cup. Because of the finite size of the vacuum chamber and the scope of the Faraday cup's linear-motion feedthrough, when the Faraday cup is at large radius, ions passing through the slot near the axis can escape. Therefore, a second disk sits behind the Faraday cup to collect stray ions. The aperture in front of the Faraday cup has been reduced in size to a diameter of $0.5 \mathrm{~cm}$ to improve spatial resolution. The copper plates will also provide a direct measurement of the total charge of the plasma.

\section{CONCLUSIONS}

By demonstrating that PTSX can create and trap plasmas that are well-behaved for hundreds of milliseconds, the opportunity exists to perform experiments in which the 


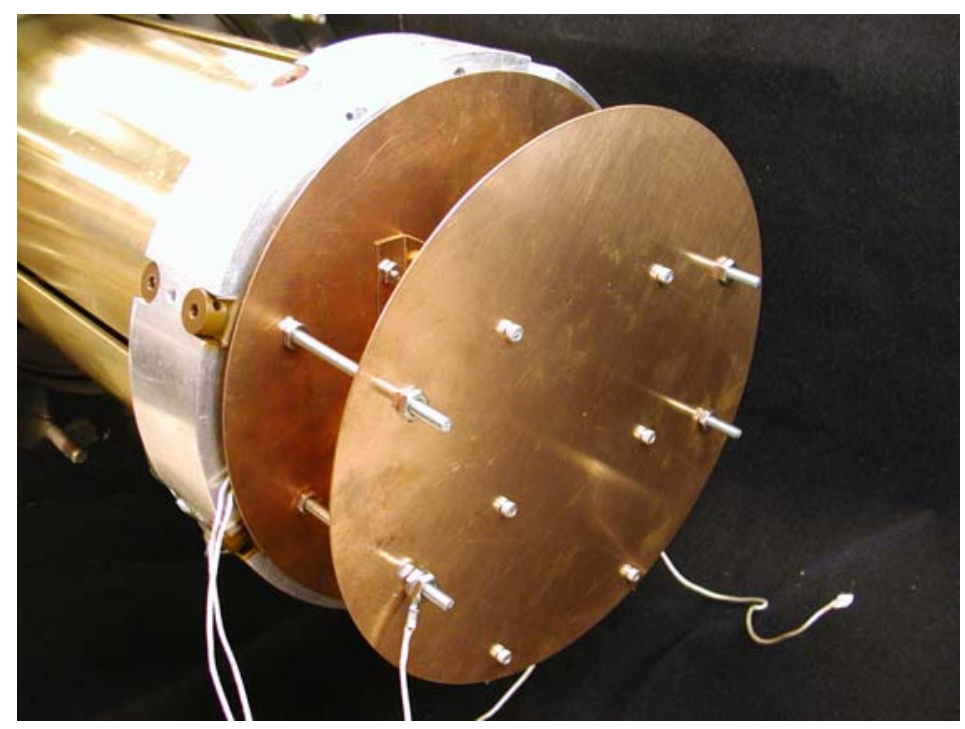

FIGURE 7. A pair of copper disks sandwiches the Faraday cup. A slot (not visible here) in the forward disk allows ions to enter the Faraday cup as the cup moves radially. The second disk acts as a backstop to catch ions that may pass through the slot but miss the Faraday cup.

voltage waveform applied to the confinement electrodes is varied to simulate a wide range of beam transport problems. Equally as important as trapping plasmas for long equivalent distances is the creation of plasmas with intensity parameter $\hat{s}=0.8$. This allows PTSX to explore nonlinear beam phenomena in regimes where intense spacecharge effects cannot be neglected. Computer simulations help to validate the PTSX results and also help to guide the design of future modifications. Improvements to the ion source and to the Faraday cup diagnostic will give finer control of the injected plasma and more precise measurements of the trapped plasma.

\section{ACKNOWLEDGMENTS}

This work was supported by the United States Department of Energy.

\section{REFERENCES}

1. Davidson, R. C., Qin, H., and Shvets, G., Phys. Plasmas, 7, 1020 (2000).

2. Okamoto, H., and Tanaka, H., Nucl. Instrum. and Methods A, 437, 178 (1999).

3. Davidson, R. C., and Qin, H., Physics of Intense Charged Particle Beams in High Intensity Accelerators, World Scientific, Singapore, 2001.

4. Reiser, M., Theory and Design of Charged Particle Beams, Wiley, New York, 1994.

5. Gilson, E. P., Davidson, R. C., Efthimion, P. C., Majeski, R., and Qin, H., Laser and Particle Beams, in press (2003).

6. Gilson, E. P., Davidson, R. C., Efthimion, P. C., Majeski, R., and Qin, H., Proceedings of the 2003 Particle Accelerator Conference, in press (2003). 
7. Celata, C. M., Bieniosek, F. M., Henestroza, E., Kwan, J. W., Lee, E. P., Logan, G., Prost, L., Seidl, P. A., Vay, J.-L., Waldron, W. L., Yu, S. S., Barnard, J. J., Callahan, D. A., Cohen, R. H., Friedman, A., Grote, D. P., Lund, S. M., Molvik, A., Sharp, W. M., Westenskow, G., Davidson, R. C., Efthimion, P., Gilson, E. P., Grisham, L. R., Majeski, R., Qin, H., Startsev, E. A., Bernal, S., Cui, Y., Feldman, D., Godlove, T. F., Haber, I., Harris, J., Kishek, R. A., Li, H., O’Shea, P. G., Quinn, B., Reiser, M., Valfells, A., Walter, M., Zou, Y., Rose, D. V., and Welch, D. R., Phys. Plasmas, 10, 2064 (2003).

8. Friedman, A., Grote, D. P., and Haber, I., Phys. Fluids B (1992). 